\title{
Transthoracic Vector Flow Imaging in Pediatric Patients with Valvular Stenosis - A Proof of Concept Study
}

\section{(우(i) $(9)$}

Authors

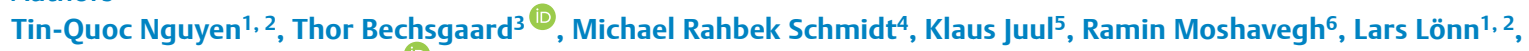
Michael Bachmann Nielsen ${ }^{1,2}{ }^{\mathbb{D}}$, Jørgen Arendt Jensen ${ }^{7}$, Kristoffer Lindskov Hansen ${ }^{1,2}$

Affiliations

1 Department of Diagnostic Radiology, Rigshospitalet Diagnostisk Center, Copenhagen, Denmark

2 Department of Clinical Medicine, University of Copenhagen, Faculty of Health and Medical Sciences, Copenhagen, Denmark

3 Department of Radiology, Odense University Hospital, Odense, Denmark

4 Rigshospitalet Hjertecentret, The Heart Center, Copenhagen, Denmark

5 Department of Pediatric Cardiology, Rigshospitalet, Copenhagen, Denmark

6 BK Medical ApS, Herlev, Denmark

7 Department of Health Technology, Technical University of Denmark, Kgs. Lyngby, Denmark

Key words

catheters, echocardiography, vector flow imaging, valvular stenosis, pressure gradient

received 17.12 .2020

accepted after revision $\quad 05.09 .2021$

published online 2021

Bibliography

Ultrasound Int Open 2021; 7: E48-E54

DOI $10.1055 / a-1652-1261$

ISSN 2199-7152

(c) 2021. The Author(s).

This is an open access article published by Thieme under the terms of the Creative Commons Attribution-NonDerivative-NonCommercial-License, permitting copying and reproduction so long as the original work is given appropriate credit. Contents may not be used for commecial purposes, or adapted, remixed, transformed or built upon. (https://creativecommons. org/licenses/by-nc-nd/4.0/)

Georg Thieme Verlag KG, Rüdigerstraße 14,

70469 Stuttgart, Germany
Correspondence

Mr. Tin-Quoc Nguyen

Department of Diagnostic Radiology

Rigshospitalet Diagnostisk Center

Blegdamsvej 9

2100 Kobenhavn

Denmark

Tel.: + 60141546

tinqnguyen@gmail.com

\section{ABSTRACT}

Purpose Continuous wave Doppler ultrasound is routinely used to detect cardiac valve stenoses. Vector flow imaging (VFI) is an angle-independent real-time ultrasound method that can quantify flow complexity. We aimed to evaluate if quantification of flow complexity could reliably assess valvular stenosis in pediatric patients.

Materials and Methods Nine pediatric patients with echocardiographically confirmed valvular stenosis were included in the study. VFI and Doppler measurements were compared with transvalvular peak-to-peak pressure differences derived from invasive endovascular catheterization.

Results Vector concentration correlated with the catheter measurements before intervention after exclusion of one outlier $(r=-0.83, p=0.01)$, whereas the Doppler method did not $(r=0.49, p=0.22)$. The change in vector concentration after intervention correlated strongly with the change in the measured catheter pressure difference $(r=-0.86, p=0.003)$, while Doppler showed a tendency for a moderate correlation $(r=0.63, p=0.07)$.

Conclusion Transthoracic flow complexity quantification calculated from VFI data is feasible and may be useful for assessing valvular stenosis severity in pediatric patients.

\section{Introduction}

Congenital heart disease affects more than 1000000 newborns yearly with an increased prevalence over the past decade [1]. Transthoracic echocardiography remains the preferred diagnostic tool for early detection of heart defects in newborns as it is a safe, wide- ly available, and cost-effective approach that enables hemodynamic assessment bedside [2]. It uses the spectral Doppler ultrasound method to measure blood velocities, which can then be calculated into pressure differences using the simplified Bernoulli equation [3]. The transvalvular pressure difference is usually calculated to 
assess the stenosis severity in pediatric patients [4]. However, the Doppler method is angle-dependent, which can result in velocity estimation errors and misclassification of disease severity [5].

Vector flow imaging (VFI) is an ultrasound method that can assess blood flow angle-independently. VFI simultaneously assesses the axial and transverse flow velocity [6] and allows for a more advanced flow interpretation than conventional Doppler ultrasound. Vascular pathology can increase flow complexity [7] and the complex flow can be visualized with VFI and further quantified as vector concentration [8].

This study used transthoracic VFI to quantify the transvalvular flow complexity in pediatric patients with aortic or pulmonary valve stenosis referred to percutaneous balloon valvuloplasty. The aim was to compare transvalvular vector concentration with invasive pressure difference measurements before and after intervention. Additionally, the peak pressure difference measured using conventional transthoracic continuous wave Doppler echocardiography was obtained. The invasive pressure catheter measurements were the reference standards, with which the two ultrasound methods were compared.

\section{Method}

\section{Study population}

Nine infants or young children (range: 20 days to 5 years, median age: 4.5 months old) referred for percutaneous balloon valvuloplasty because of congenital aortic or pulmonary valve stenosis were invited to participate in the study. Informed consent was obtained from the parents. The local ethics committee waived approval since ultrasound is a routine examination, and VFI findings did not diagnose or alter the treatment of any participants (protocol no. 17020259).

During catheterization in one patient (patient no. 8), a supravalvular stenosis was revealed in proximity to the aortic valve instead of a valvular stenosis. Balloon dilation was attempted on the stenotic segment without success, and the patient was subsequently referred for surgery. The post-procedural Doppler and vector concentration datasets were still obtained, and patient no. 8 remained included in the study.

\section{Vector flow imaging}

All VFI sequences were acquired using a modified commercial ultrasound scanner (bk5000, BK Medical, Herlev, Denmark) and a linear transducer (8L2, BK Medical, Herlev, Denmark). The VFI method used in this study is currently unavailable on dedicated transthoracic cardiac transducers, but transthoracic scanning using linear transducers has been shown to be feasible for pediatric patients $[9,10]$. The scanner was set to VFI mode with a color wheel to indicate the flow direction and magnitude of velocity in 360 degrees, - Fig. 1. During anesthesia and before valvuloplasty, the transducer was positioned parasternal and readjusted to optimize the view of the transvalvular blood flow. The VFI color box would ideally include the midstenotic and adjacent pre- and post-stenotic flow segments, > Fig. 2. When an optimal image could not be acquired, e. g., due to costal shadowing or pulmonary overlay, the midstenotic flow was prioritized. After valvuloplasty, VFI sequences were acquired once more before anesthesia was terminated. VFI was obtained at a frame rate of 30 frames per second. Data were processed on an offline workstation using an graphical user interface built inhouse in MATLAB (MathWorks, Natick, MA, USA) [11]. A region of interest (ROI) was manually selected inside the interface at peak

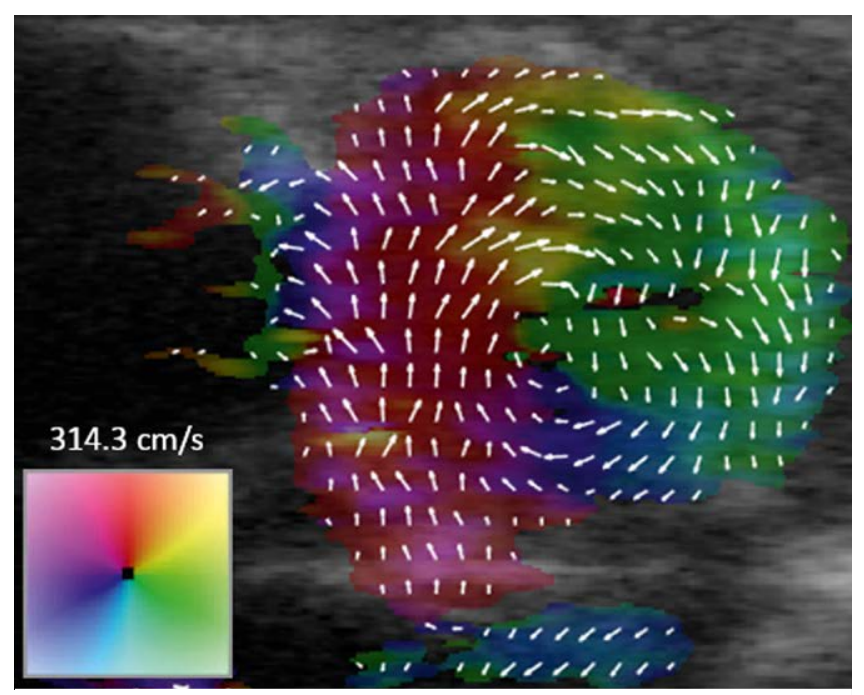

- Fig. 1 Short-axis view of the pulmonary artery after intervention using vector flow imaging. The blood flow direction spans all 360 degrees. Here, blood flow is moving in a clockwise direction.

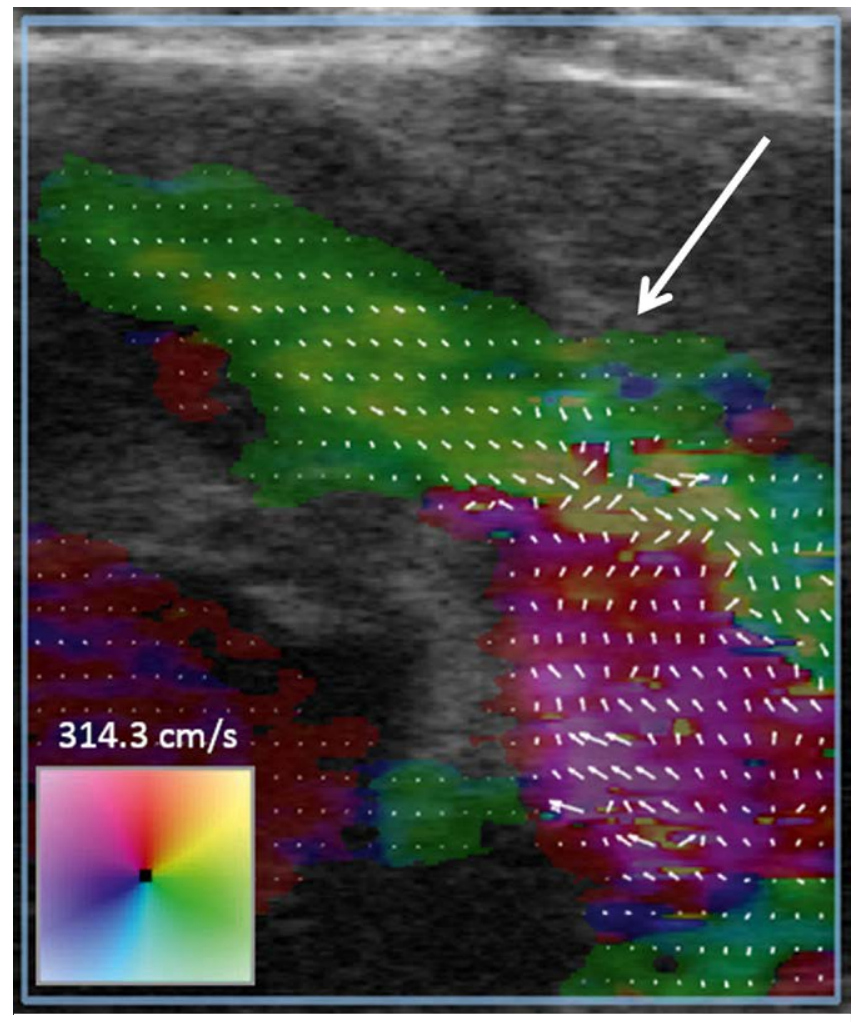

- Fig. 2 Long-axis view of the pulmonary artery after intervention using vector flow imaging. During systole, blood leaves the right ventricle (top-left), passes through the pulmonary valve (arrow), and enters the pulmonary artery (bottom right). 
systole. The ROIs extended approximately one vessel diameter upstream and downstream from the stenosis while still staying within the boundaries of the vessel. An example of a drawn ROI can be seen in $>$ Fig. 3 . The output was a parameter called vector concentration; a number between zero and one was used to indicate a range of flow from highly complex to completely laminar [8]. A mean vector concentration was calculated from 10 systoles with a new ROI being defined for each systole to accommodate for any change in valve position. A more thorough explanation of the calculation has been described in previous papers [8, 12].

\section{Transthoracic echocardiography}

A clinical examination and transthoracic echocardiography were performed within 24 hours prior to balloon valvuloplasty. Echocardiography was done by experienced pediatric cardiologists using a commercially available ultrasound scanner (Philips EPIQ 7C, Philips Ultrasound, Inc., Bothell-Everett Hwy, Bothell, WA, USA) with dedicated cardiac transducers. Using the imaging plane with the lowest possible angle error, the instantaneous peak systolic pressure difference across the valve was calculated using the simplified Bernoulli equation [3], see > Fig. 4. Images and calculations were stored in the IntelliSpace Cardiovascular software suite
(Philips Medical Systems Nederland B.V., Veenpluis, The Netherlands). A repeat transthoracic echocardiography was performed the day after balloon valvuloplasty with estimation of the post-procedural transvalvular pressure difference.

\section{Catheterization}

During anesthesia, left or right heart catheterization was conducted via direct puncture of the right common femoral artery or vein below the inguinal ligament. Using a 4F-6F sheath, a multipurpose catheter on a Terumo wire was maneuvered to the heart under fluoroscopy guidance and through the stenotic valve. The multipurpose catheter was subsequently replaced by a pigtail catheter over the wire. In the case of pulmonary valvular stenosis, the pulmonary artery pressure was measured before the pigtail catheter was pulled back through the valve to measure the pre-stenotic pressure. Intervention was performed on mild stenoses if assessed by clinicians as relevant. Angiography confirmed the presence of a valvular stenosis and the absence of a sub- and supravalvular obstruction.

Balloon valvotomy was performed using compliant balloons (Tyshak, Numed Inc., Hopkinton, NY, USA) with a diameter according to the standard guidelines (100-130\% of annular diameter for pulmonary and $80-100 \%$ in aortic valves) [13]. Post-valvotomy angi-
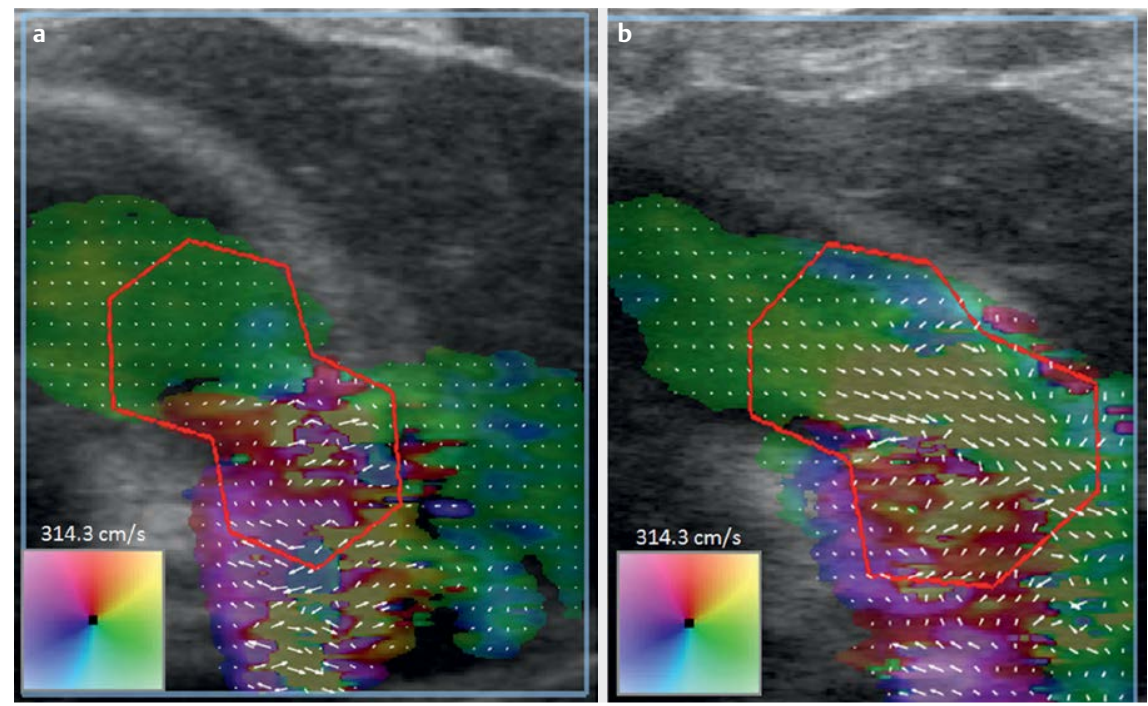

- Fig. 3 Vector flow imaging of a pulmonary valve stenosis before (a) and after (b) treatment. Vector concentration was calculated inside an ROI. In this patient, vector concentration increased from 0.40 to 0.51 .
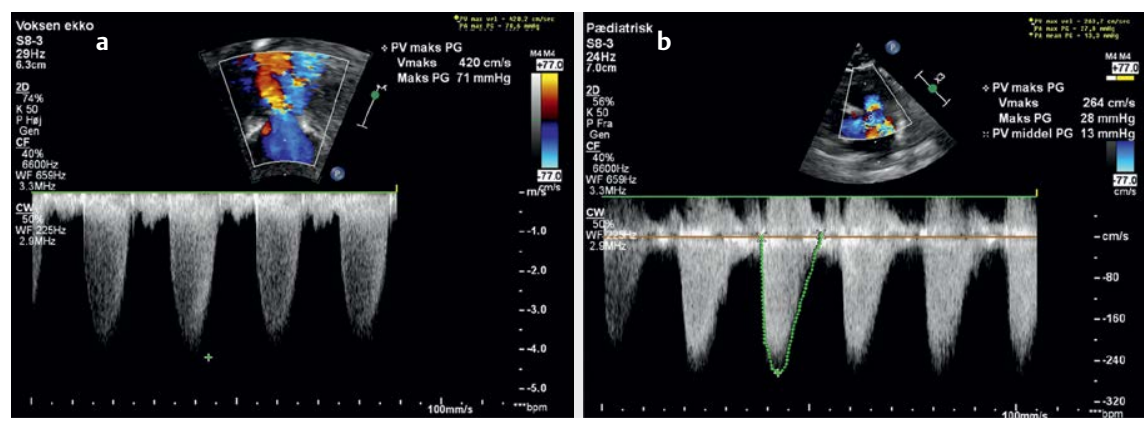

-Fig. 4 Transthoracic echocardiography of a pulmonary valve stenosis before (a) and after (b) intervention (same patient as in > Fig. 3). 
ography checked for the absence of rupture and valve regurgitation followed by post-procedural pressure measurement in the aorta/pulmonary artery and in the left/right ventricle. If a significant residual pressure difference across the valve was noted, valvotomy was done with a larger balloon.

\section{Statistical analysis}

Data were analyzed with descriptive statistics. Pearson correlation coefficients were calculated to assess the linear relationship between the pressure catheter measurements and both ultrasound methods. A p-value $<0.05$ was considered statistically significant. All statistical analyses were performed in the statistical programming software, R (R Foundation for Statistical Computing, Vienna, Austria).

\section{Results}

The transvalvular pressure differences measured with Doppler and pressure catheter before and after intervention as well as the mean transvalvular vector concentration are listed in $\mathbf{T a b l e ~} \mathbf{1}$. The differences between before and after intervention are summarized in $>$ Table 2.

Scatterplots of the pooled data points (before + after) are illustrated in $\triangleright \mathbf{F i g}$. 5. The patient with no valvular stenosis had a higher vector concentration compared with the others. Data were revisited for one outlier with a noticeably high vector concentration after intervention (patient no. 5), but no satisfactory explanation was found.

Neither vector concentration $(r=-0.63, p=0.07)$ nor continuous wave Doppler ultrasound pressure differences $(r=0.48$, $p=0.19$ ) showed a significant correlation with the catheter measurements before intervention. However, exclusion of the patient without valvular stenosis resulted in a strong correlation coefficient for vector concentration $(r=-0.83, p=0.01)$, but no change for the Doppler method $(r=0.49, p=0.22)$. After intervention, the correlation was no longer significant for vector concentration $(r=-0.49, p=0.18)$ or Doppler $(r=0.16, p=0.69)$.
The change in vector concentration after treatment showed a strong correlation with the change in pressure difference measured with a pressure catheter $(r=-0.86, p=0.003)$, while a tendency for a correlation was found for the Doppler method ( $r=0.63, p=0.07$ ).

\section{Discussion}

This study demonstrated the transvalvular vector concentration parameter to correlate strongly with catheter pressure differences before intervention after exclusion of an outlier without stenosis. Brandt et al. [14] and Hansen et al. [12] reported strong correlations between vector concentration and stenosis severity in carotid and femoral artery stenoses, and Saris et al. [7] found increased flow complexity in diseased carotid arteries. However, their reference methods differed from this study, and the reported correlations may not be comparable. Cardiac catheterization is still regard-

\begin{tabular}{|c|c|c|c|c|}
\hline Patient \# & $\begin{array}{l}\text { Stenosis } \\
\text { location }\end{array}$ & $\begin{array}{l}\Delta \text { Pressure } \\
\text { catheter } \\
(\mathrm{mmHg})\end{array}$ & $\begin{array}{l}\Delta \text { Doppler } \\
(\mathrm{mmHg})\end{array}$ & $\begin{array}{l}\Delta \text { Vector } \\
\text { concentra- } \\
\text { tion }\end{array}$ \\
\hline 1 & Pulm. & -7 & -0.2 & +0.03 \\
\hline 2 & Ao. & -46 & -44 & +0.13 \\
\hline 3 & Pulm. & -2 & +2 & +0.01 \\
\hline 4 & Pulm. & -5 & -44 & +0.02 \\
\hline 5 & Pulm. & -64 & -67.4 & +0.46 \\
\hline 6 & Pulm. & -6 & -43 & +0.12 \\
\hline 7 & Pulm. & -20 & +8.6 & +0.14 \\
\hline 8 & Ao. & -1 & -10.5 & -0.08 \\
\hline 9 & Pulm. & -13 & -4.8 & +0.14 \\
\hline
\end{tabular}

- Table 1 Catheter pressure differences, continuous wave Doppler ultrasound pressure differences, and vector concentration before and after treatment.

\begin{tabular}{|c|c|c|c|c|c|c|c|}
\hline \multirow[b]{2}{*}{ Patient \# } & \multirow[b]{2}{*}{ Stenosis location } & \multicolumn{2}{|c|}{ Pressure catheter $(\mathrm{mmHg})$} & \multicolumn{2}{|c|}{ Doppler (mmHg) } & \multicolumn{2}{|c|}{ Vector concentration } \\
\hline & & Before & After & Before & After & Before & After \\
\hline 1 & Pulm. & 15 & 8 & 24.2 & 24 & 0.48 & 0.51 \\
\hline 2 & Ao. & 57 & 11 & 62 & 18 & 0.37 & 0.50 \\
\hline 3 & Pulm. & 18 & 16 & 57 & 59 & 0.49 & 0.51 \\
\hline 4 & Pulm. & 40 & 35 & 78 & 34 & 0.39 & 0.41 \\
\hline 5 & Pulm. & 75 & 11 & 76.2 & 9 & 0.33 & 0.79 \\
\hline 6 & Pulm. & 20 & 14 & 70.8 & 28 & 0.40 & 0.51 \\
\hline 7 & Pulm. & 35 & 15 & 82.4 & 91 & 0.37 & 0.51 \\
\hline 8 & Ao. & 13 & 12 & 58.5 & 48 & 0.82 & 0.74 \\
\hline 9 & Pulm. & 35 & 22 & 50.3 & 45.5 & 0.41 & 0.55 \\
\hline
\end{tabular}



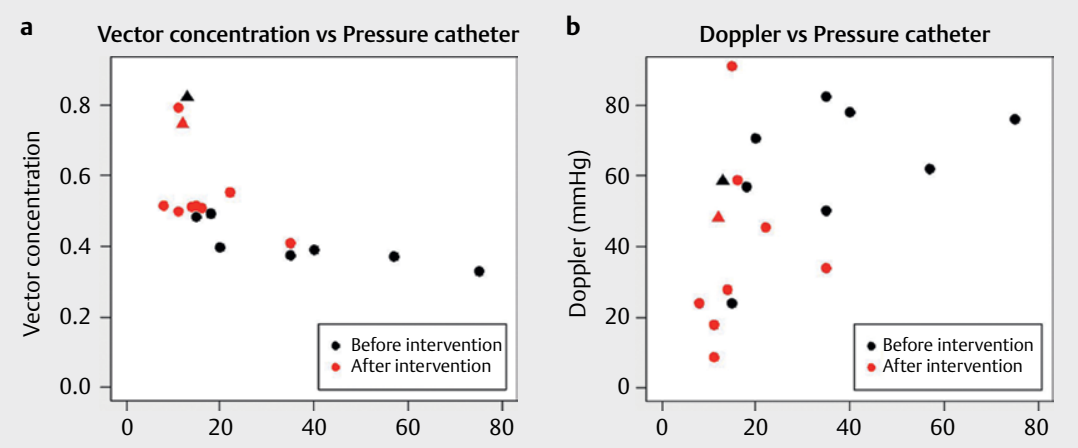

- Fig. 5 Vector concentration (a) and Doppler pressure differences (b) plotted against pressure differences measured with a pressure catheter. One patient had no valvular stenosis and is marked with triangles $(\mathbf{\Lambda})$.

ed as the "gold standard" for assessing congenital heart disease, and invasive pressure difference measurement can help assess the risk of the disease and reveal a need for intervention [15]. However, the procedure has both short- and long-term risks, and noninvasive approaches, such as echocardiography, should be considered first $[15,16]$. A noninvasive approach capable of correctly identifying patients needing interventional treatment could reduce the number of unnecessary procedures.

The Doppler results differed from previous comparison studies of Doppler and catheter measurements. These studies reported mostly strong linear correlations across stenotic aortic valves [17-19], whereas the two ultrasound methods did not correlate with the catheter method in this study. A high Doppler pressure difference was measured in one patient despite the absence of valvular stenosis, likely due to the vicinity of a downstream supravalvular stenosis. Continuous wave Doppler ultrasound allows the user to measure higher velocities than conventional pulsed Doppler ultrasound without aliasing of the spectrum, but obscures the exact depth of the measured velocity [20]. The time delay between the Doppler and catheter measurements might also have influenced the results, since partial valve recoil might have interfered with the post-procedural Doppler examination [21]. Other limitations are the small sample size as well as the inherent difference when comparing instantaneous and peak-to-peak pressure differences [22].

Doppler pressure differences are reported to overestimate catheter measurements, and the discrepancy is commonly attributed to the lack of correction for pressure recovery $[17,18]$. However, correction for pressure recovery may be unnecessary [19]. Also, Doppler pressure differences are calculated from peak velocities, thereby making them instantaneous pressure differences. Meanwhile, the catheter pressure differences in this study were measured peak-to-peak using the pull-back method. Instantaneous pressure differences are fundamentally different from peak-to-peak pressure differences and will always be higher [22]. Therefore, a discrepancy between the two methods will always exist.

Like conventional spectral Doppler ultrasound, VFI is based on pulsed ultrasound emissions, and high blood flow velocities cause aliasing artifacts. The artifacts distort the velocity information and prevent flow velocity estimation with Doppler [23]. Similarly, aliased flow reverses the velocity vectors with VFI and impedes velocity estimation. However, the uniformity of the reversed flow re- mains largely the same and vector concentration estimation may still be feasible [12,24].

Unlike the spectral Doppler technique, VFI is not angle-dependent. Aortic stenoses and bicuspid aortic valves displace flow away from the center of the ascending aorta $[25,26]$. This complicates peak systolic velocity measurement with Doppler, since the velocity error increases with the angle error and correlates with the magnitude of displacement [25]. The Doppler-catheter ratio can decrease up to $80 \%$ as the jet gets increasingly eccentric [27]. VFI displays the velocity field on the monitor and eccentric flow is easily detected [28]. Furthermore, VFI is more accurate for peak systolic velocities in the common carotid artery than spectral Doppler ultrasound [29]. As such, VFI may be a good alternative to conventional Doppler ultrasound for stenosis assessment, as it obtains and utilizes more of the available flow information and is angle-independent, more accurate, and less operator-dependent.

The first studies on cardiac VFI listed limited penetration depth as a drawback for the technique, and cardiac flow assessment was then performed intraoperatively directly on the heart to preserve accuracy [28]. However, transthoracic cardiac flow assessment has since been demonstrated to be feasible in pediatric patients $[9,10]$. We managed to produce usable vector field images on a 4 years and 9 months old patient, indicating that future studies may include even older pediatric patients.

This study had several limitations. First, the study included only nine patients and the amount of data obtained was limited. Congenital aortic stenosis and pulmonary valve stenosis are rare diseases found in only 1.9 and 5.5 per 10000 live births, respectively [1]. However, we recognize the need for a larger study sample for more conclusive statements. Therefore, this study is a proofof-concept and should be interpreted as such. Another limitation is that VFI visualizes flow movements in a 2D plane. This is better than conventional Doppler ultrasound that only measures velocities in 1D. Out-of-plane flow is still omitted in the vector concentration analysis. Blood flow in the ascending aorta is known to be helical and vortical $[26,28]$, and essential flow information is lost with a 2D VFI technique. A 3D VFI approach already exists [30] and would likely have resulted in better estimation of the transvalvular flow and turbulence. The time lag between Doppler measurements and the intervention is also a limitation. Additionally, the Doppler examination was not performed during anesthesia, which could 
have increased cardiac output and hindered optimal data acquisition due to patient movement. Recoil of the stenotic area may have occurred during the post-procedural Doppler examination, which would result in an altered flow environment, ultimately impacting the correlation with invasively measured pressure differences [21]. The pediatricians rotated their functions daily and the transthoracic echocardiography before and after intervention was not necessarily performed by the same person. Interobserver variability may have influenced the estimated changes in Doppler velocities. Data were analyzed by only one investigator (TN) without assessment of inter- and intraobserver variability. Therefore, we cannot dismiss that the small decreases or increases in vector concentration observed in this study were due to intraobserver variability. Investigating the inter- and intraobserver variability of vector concentration estimation should be the topic of a future study. Lastly, we used an in-house build toolbox to analyze our data and calculate the vector concentration. The method is published in peer-reviewed journals $[8,12]$ but is not yet commercially available.

\section{Conclusion}

The vector concentration and Doppler methods did not correlate with the transvalvular pressure differences measured using direct catheterization in this study of nine pediatric patients. The change in vector concentration following treatment correlated strongly with the catheter pressure differences. This proof-of-concept study indicated that transvalvular flow complexity may be quantified transthoracically with VFI. Larger studies comparing VFI with conventional spectral Doppler ultrasound and invasive pressure catheters are needed to verify the findings in the present study.

\section{Acknowledgements}

The authors would like to thank Rikke Karlsen, Jesper Reimers, Jesper Steensberg, Jim Dodd, and Lars Idorn from the Department of Pediatric Cardiology at Rigshospitalet, Copenhagen, for helping with the recruitment process and cardiac ultrasound.

\section{Conflict of Interest}

Ramin Moshavegh is employed at BK Medical Aps. Jørgen Arendt Jensen developed and patented the vector flow imaging technique.

\section{References}

[1] Liu Y, Chen S, Zühlke L et al. Global birth prevalence of congenital heart defects 1970-2017: Updated systematic review and meta-analysis of 260 studies. Int J Epidemiol 2019; 48: 455-463. doi:10.1093/ije/ dyz009

[2] Singh Y. Echocardiographic Evaluation of Hemodynamics in Neonates and Children. Front Pediatr 2017; 5: 201. doi:10.3389/ fped.2017.00201

[3] Yoganathan AP, Cape EG, Sung HW et al. Review of hydrodynamic principles for the cardiologist: Applications to the study of blood flow and jets by imaging techniques. J Am Coll Cardiol 1988; 12: 13441353
[4] Singh GK. Congenital Aortic Valve Stenosis. Children (Basel) 2019; 6. doi:10.3390/children6050069

[5] Ringle A, Castel A-L, Le Goffic C et al. Prospective assessment of the frequency of low gradient severe aortic stenosis with preserved left ventricular ejection fraction: Critical impact of aortic flow misalignment and pressure recovery phenomenon. Arch Cardiovasc Dis 2018; 111: 518-527. doi:10.1016/j.acvd.2017.11.004

[6] Jensen JA, Munk P. A new method for estimation of velocity vectors. IEEE T Ultrason Ferr 1998; 45: 837-851. doi:10.1109/58.677749

[7] Saris AECM, Hansen HHG, Fekkes S et al. In Vivo Blood Velocity Vector Imaging Using Adaptive Velocity Compounding in the Carotid Artery Bifurcation. Ultrasound Med Biol 2019; 45: 1691-1707. doi:10.1016/j. ultrasmedbio.2019.03.008

[8] Pedersen MM, Pihl M], Haugaard P et al. Novel flow quantification of the carotid bulb and the common carotid artery with vector flow ultrasound. Ultrasound Med Biol 2014; 40: 2700-2706. doi:10.1016/j. ultrasmedbio.2014.06.001

[9] Collins RT II, Laughlin ME, Lang SM et al. Real-time transthoracic vector flow imaging of the heart in pediatric patients. Prog Pediatr Cardiol 2019; 53: 28-36. doi:10.1016/j.ppedcard.2019.02.003

[10] Hansen KL, Juul K, Møller-Sørensen H et al. Pediatric Transthoracic Cardiac Vector Flow Imaging - A Preliminary Pictorial Study. Ultrasound International Open 2019; 5: E20-E26. doi:10.1055/a-0656-5430

[11] Moshavegh R, Martins B, Hansen K et al. Hybrid segmentation of vessels and automated flow measures in in-vivo ultrasound imaging. Proc. IEEE Ultrason.Symp. 2016; 1-4. doi:10.1109/ULTSYM.2016.7728656

[12] Hansen KL, Hansen PM, Ewertsen C et al. Vector Flow Imaging Compared with Digital Subtraction Angiography for Stenosis Assessment in the Superficial Femoral Artery - A Study of Vector Concentration, Velocity Ratio and Stenosis Degree Percentage. Ultrasound International Open 2019; 5: E53-E59. doi:10.1055/a-0853-2002

[13] Syamasundar Rao P. Balloon Valvuloplasty and Angioplasty in Pediatric Practice. Pediatr Therapeut 2011; 01. doi:10.4172/21610665.1000e103

[14] Brandt AH, Nguyen T-Q, Gutte H et al. Carotid Stenosis Assessment with Vector Concentration before and after Stenting. Diagnostics 2020; 10: 420. doi:10.3390/diagnostics 10060420

[15] Feltes TF, Bacha E, Beekman RH et al. Indications for Cardiac Catheterization and Intervention in Pediatric Cardiac Disease. Circulation 2011; 123: 2607-2652. doi:10.1161/ CIR.0b013e31821b1f10

[16] Schneuer F], Bentley JP, Davidson AJ et al. The impact of general anesthesia on child development and school performance: A population-based study. Pediatric Anesthesia 2018; 28: 528-536. doi:10.1111/pan. 13390

[17] Baumgartner H, Stefenelli T, Niederberger J et al. „Overestimation“ of catheter gradients by Doppler ultrasound in patients with aortic stenosis: A predictable manifestation of pressure recovery. J Am Coll Cardiol 1999; 33: 1655-1661

[18] VanAuker MD, Hla A, Meisner JS et al. Simultaneous Doppler/catheter measurements of pressure gradients in aortic valve disease: $A$ correction to the Bernoulli equation based on velocity decay in the stenotic jet. J Heart Valve Dis 2000; 9: 291-298

[19] Yamazawa H. Accuracy of the Doppler-derived pressure gradient in pediatric patients with aortic valvular stenosis: Is the correction for pressure recovery necessary? Hokkaido Igaku Zasshi 2010; 85: 225-231 
[20] Reeder GS, Currie PJ, Hagler DJ et al. Use of Doppler Techniques (Continuous-Wave, Pulsed-Wave, and Color Flow Imaging) in the Noninvasive Hemodynamic Assessment of Congenital Heart Disease. Mayo Clin Proc 1986; 61: 725-744. doi:10.1016/S00256196(12)62774-8

[21] Pihkala J, Nykanen D, Freedom RM et al. Interventional cardiac catheterization. Pediatr Clin N Am 1999; 46: 441-464. doi:10.1016/ S0031-3955(05)70128-0

[22] Nishimura RA, Carabello BA. Hemodynamics in the Cardiac Catheterization Laboratory of the 21st Century. Circulation 2012; 125 : 2138-2150. doi:10.1161/CIRCULATIONAHA.111.060319

[23] Terslev L, Diamantopoulos AP, Døhn UM et al. Settings and artefacts relevant for Doppler ultrasound in large vessel vasculitis. Arthritis Res Ther 2017; 19: 1-10. doi:10.1186/s13075-017-1374-1

[24] Nguyen T-Q, Traberg MS, Olesen JB et al. Flow Complexity Estimation in Dysfunctional Arteriovenous Dialysis Fistulas using Vector Flow Imaging. Ultrasound Med Biol 2020; 46: 2493-2504. doi:10.1016/j. ultrasmedbio.2020.05.021

[25] Adriaans BP, Westenberg JJM, van Cauteren YJM et al. Clinical assessment of aortic valve stenosis: Comparison between 4D flow MR and transthoracic echocardiography. J Magn Reson Imaging 2020; 51: 472-480. doi:10.1002/jmri.26847
[26] Garcia J, Barker A], Markl M. The Role of Imaging of Flow Patterns by 4D Flow MRI in Aortic Stenosis. JACC Cardiovasc Imag 2019; 12: 252-266. doi:10.1016/j.jcmg.2018.10.034

[27] Niederberger J, Schima H, Maurer G et al. Importance of Pressure Recovery for the Assessment of Aortic Stenosis by Doppler Ultrasound. Circulation 1996; 94: 1934-1940. doi:10.1161/01.CIR.94.8.1934

[28] Hansen KL, Møller-Sørensen H, Kjaergaard J et al. Intra-Operative Vector Flow Imaging Using Ultrasound of the Ascending Aorta among 40 Patients with Normal, Stenotic and Replaced Aortic Valves. Ultrasound Med Biol 2016; 42: 2414-2422. doi:10.1016/j.ultrasmedbio.2016.06.009

[29] Brandt AH, Hansen KL, Ewertsen C et al. A Comparison Study of Vector Velocity, Spectral Doppler and Magnetic Resonance of Blood Flow in the Common Carotid Artery. Ultrasound Med Biol 2018; 44: 1751-1761. doi:10.1016/j.ultrasmedbio.2018.05.002

[30] Holbek S, Hansen KL, Bouzari H et al. Common Carotid Artery Flow Measured by 3-D Ultrasonic Vector Flow Imaging and Validated with Magnetic Resonance Imaging. Ultrasound Med Biol 2017; 43: 2213-2220. doi:10.1016/j.ultrasmedbio.2017.06.007 